EXTENDED REPORT

\title{
Retinal toxicity of intravitreal tenecteplase in the rabbit
}

\author{
S A Rowley, S Vijayasekaran, P K Yu, I L McAllister, D-Y Yu
}

Br J Ophthalmol 2004;88:573-578. doi: 10.1136/bjo.2003.027466

\begin{abstract}
Aim: To investigate the retinal toxicity of intravitreal injection of a novel fibrinolytic tenecteplase in rabbit eyes.

Methods: Tenecteplase (25-350 $\mu \mathrm{g}$ in $0.1 \mathrm{ml} \mathrm{BSS})$ was injected into the vitreous cavity of normal rabbit eyes. Control (fellow) eyes received $0.1 \mathrm{ml}$ of BSS. One day, 1 week, and 2 months post-injection, the eyes were examined by slit lamp biomicroscopy, indirect ophthalmoscopy, and electroretinography, and then harvested for histopathological examination.

Results: No evidence of retinal toxicity was seen with tenecteplase doses up to and including $50 \mu \mathrm{g}$. At a dose of $150 \mu \mathrm{g}$ ophthalmoscopy was normal, but histology showed mild retinal damage in the inner nuclear layer and electroretinography showed a temporary reduction in B-wave amplitude. At doses of $200 \mu \mathrm{g}$ and above, there was evidence of retinal toxicity on electroretinography, ophthalmoscopy, and histology. Ophthalmoscopic findings included vitreal fibrosis, retinal necrosis and tractional retinal detachment and light microscopy revealed necrosis of retinal pigment epithelium and other retinal layers. Damage was centred around the injection site but was more widespread with the higher doses.

Conclusion: A dose of $50 \mu \mathrm{g}$ tenecteplase appears safe for intravitreal injection in the rabbit. Tenecteplase could have potential applications in the treatment of submacular haemorrhage and retinal vein occlusion.
\end{abstract}

See end of article for authors' affiliations

Correspondence to:

Professor Dao-Yi Yu,

Centre for Ophthalmology

and Visual Science, The

University of Western

Australia, Nedlands, WA

6009, Australia;

dyyu@cyllene.uwa.edu.au

Accepted for publication 1 September 2003

$\mathrm{T}$ issue plasminogen activator (tPA) is a recombinant plasminogen activator which binds to the fibrin component of thrombus and converts thrombus bound plasminogen to plasmin which initiates local fibrinolysis. Although primarily used in the management of acute myocardial infarction, it has also been used for various indications in the field of ophthalmology. These include dissolution of fibrin from the surface of intraocular lenses, ${ }^{1}$ accelerating the resolution of post-vitrectomy fibrin, ${ }^{2}$ treatment of central retinal vein occlusion with local $^{3}$ and systemic treatment, ${ }^{4}$ and facilitating clearance of submacular haemorrhage. ${ }^{5}$

Shortcomings of tPA in the management of acute myocardial infarction include short half life necessitating infusion rather than a bolus injection ${ }^{6}$ and this has led to the development of modified tPA compounds such as tenecteplase. Compared to tPA, tenecteplase has a longer half life and greater fibrin specificity, and so has potentially greater efficacy in thrombus dissolution. ${ }^{7}$ This could provide significant advantages in ophthalmic applications.

The results in animal studies of intravitreal injection of tPA in concentrations greater than $25 \mu \mathrm{g} / 0.1 \mathrm{ml}$ have implied that use of this dose of tPA in humans would be unsafe. ${ }^{89}$ As tenecteplase may have a better outcome in ophthalmic use, we therefore investigated the retinal toxicity of intravitreal injections of various doses of tenecteplase using slit lamp examination, indirect ophthalmoscopy, electroretinography (ERG), and light microscopy.

\section{MATERIALS AND METHODS}

The tenecteplase used in this study was the commercially available preparation and was donated by the manufacturer (Metalyse; Boehringer Ingelheim, NSW, Australia). The undiluted drug after reconstitution had a concentration of $5 \mathrm{mg} / \mathrm{ml}$. To establish the toxicity profile of the vehicle, a stock solution was prepared consisting of L-arginine $(52.2 \mathrm{mg} / \mathrm{ml})$, polysorbate $20(0.4 \mathrm{mg} / \mathrm{ml})$, and phosphoric acid $85 \% \quad(16 \mathrm{mg} / \mathrm{ml})$ (product information, Boehringer Ingelheim). As the stability of cryopreserved solutions of tenecteplase has not been established, the drug was freshly

reconstituted and diluted to the appropriate concentration each time surgery was performed.

\section{Surgery}

The rabbits were anaesthetised using an intramuscular combination of ketamine hydrochloride $(35-50 \mathrm{mg} / \mathrm{kg}$ ) and xylazine hydrochloride $(3-5 \mathrm{mg} / \mathrm{kg}$ ). The pupils were dilated with $2.5 \%$ phenylephrine hydrochloride and $1 \%$ atropine. Following baseline ERG, the retina was examined before injection using a planoconcave contact lens and operating microscope. A 30 gauge needle was introduced into the vitreous cavity $2 \mathrm{~mm}$ posterior to the nasal limbus and under direct vision with the contact lens, the needle tip (bevel anterior) was positioned just above the retinal surface 1.5 disc diameters below the inferior edge of the optic disc. A volume of $0.1 \mathrm{ml}$ of the test solution was then administered. The needle was held in place for 10-15 seconds after injection before withdrawing to prevent reflux from the entry site. The central retinal artery was observed to be patent after each injection. The fellow eye of each animal was used as a control and injected with $0.1 \mathrm{ml}$ of BSS. Chloramphenicol ointment 1\% (Chirosig, Sigma Pharmaceuticals Ltd, Victoria, Australia) was applied to the eyes immediately after surgery.

A total of 35 New Zealand White rabbits weighing 2-4 kg, were used in this study. To establish the dose levels to be tested for the main study a pilot study using 13 of the rabbits was conducted and the tenecteplase was diluted in sterile balanced salt solution (BSS; Cytosol Ophthalmics Inc, Lenoir, USA) to give doses of $25,50,100,200,250$, and $350 \mu \mathrm{g}$. As part of this pilot study, vehicle equivalents of all these tenecteplase doses were also prepared as previous work has established that the toxicity of tPA is caused by the vehicle. $^{8}$

After the dose levels had been established, the main study was performed using tenecteplase doses of 50, 150, 200, and $250 \mu \mathrm{g}$. In all, 22 rabbits were used for this study, six being used for $50 \mu \mathrm{g}$ and $250 \mu \mathrm{g}$ and five being used for $150 \mu \mathrm{g}$ and $200 \mu \mathrm{g}$. 


\section{Clinical follow up}

All the eyes were examined by slit lamp biomicroscopy, and indirect ophthalmoscopy 1 day, 1 week, and 2 months postinjection. ERG and intraocular pressure measurement (Tonopen, Mentor Ophthalmics, MA, USA) were also recorded at each examination.

\section{Electroretinography}

Photopic ERG recording was carried out on anaesthetised rabbits using a Nicolet Compact Four electroretinography suite and Ganzfeld stimulation (Nicolet Biomedical Instruments, WI, USA) with background light set to level 2. The reference electrodes were made of silver wires electroplated with chloride. The electrodes were threaded into the scalp and lateral canthus of the rabbit head.

The corneal electrode was a contact lens electrode (ERGjet, Universo sa, Switzerland). Amplifier bandpass was 1$1000 \mathrm{~Hz}$, and the stimulus interval was 0.9 seconds. Each reported ERG is the average of 32 consecutive flashes. Using four increasing stimulus intensities of $0.5,0.75,1.0$, and 1.50 $\log$ units, four averaged ERGs were recorded for each eye at every time point. Data were recorded at four time points; immediately before the injection of tenecteplase, then 1 day, 1 week, and 2 months after the injection. B-wave amplitude was measured from the A-wave trough for each recording (fig 1).

\section{Histology}

After final examination by ophthalmoscopy and electroretinography, the animals were killed with lethal injection of pentobarbitone and the eyes enucleated. Each eye had a small slit made in the region of the pars plana and was immediately placed in $2.5 \%$ glutaraldehyde in $0.1 \mathrm{M}$ phosphate buffer. After 30 minutes the anterior segment was removed. Three days later, the eye cup was vertically bisected. One half was dehydrated in graded series of ethanol and processed in paraffin (Paramat) (BDH, England). Several pieces of tissue from the other half, approximately $2 \times 3 \mathrm{~mm}$ were excised from the optic disc, medullary ray, inferior and superior retina, and from the cornea and processed for epoxy resin (Durcapan ACM, Fluka AG, Switzerland). The pieces were first post-fixed in osmium tetroxide, then dehydrated in graded series of ethanol, infiltrated with and embedded in epoxy resin. Sections of $4 \mu \mathrm{m}$ paraffin and $1 \mu \mathrm{m}$ epoxy were cut and stained. Paraffin sections were stained with haematoxylin and eosin, and epoxy sections with toluidine blue for light microscopic examination.

\section{Statistics}

Statistical analysis of intraocular pressure and ERG data was performed using paired $t$ test. B-wave amplitudes of different time points after tenecteplase administration were expressed as a percentage of that before tenecteplase administration to minimise the effect of inter-animal variation. Statistical significance was determined as $\mathrm{p}$ value equal to or less than 0.05 .

All experimental procedures were carried out in accordance with the animal experimentation ethics committee of the University of Western Australia.

\section{RESULTS}

\section{Clinical follow up}

\section{Tenecteplase and control eyes injections}

None of the 35 control eyes injected with $0.1 \mathrm{ml}$ BSS developed posterior segment inflammation or damage. Two of the control eyes had mild anterior chamber activity on day 1 , which settled after 1 week.

The eye injected with $25 \mu \mathrm{g}$ tenecteplase showed no sign of anterior or posterior chamber inflammation. Of the eight eyes
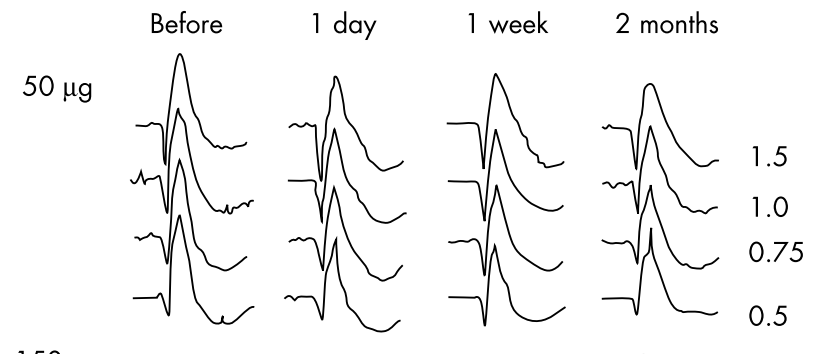

$150 \mu \mathrm{g}$
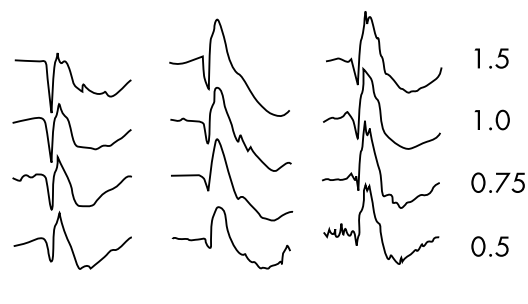

$200 \mu \mathrm{g}$
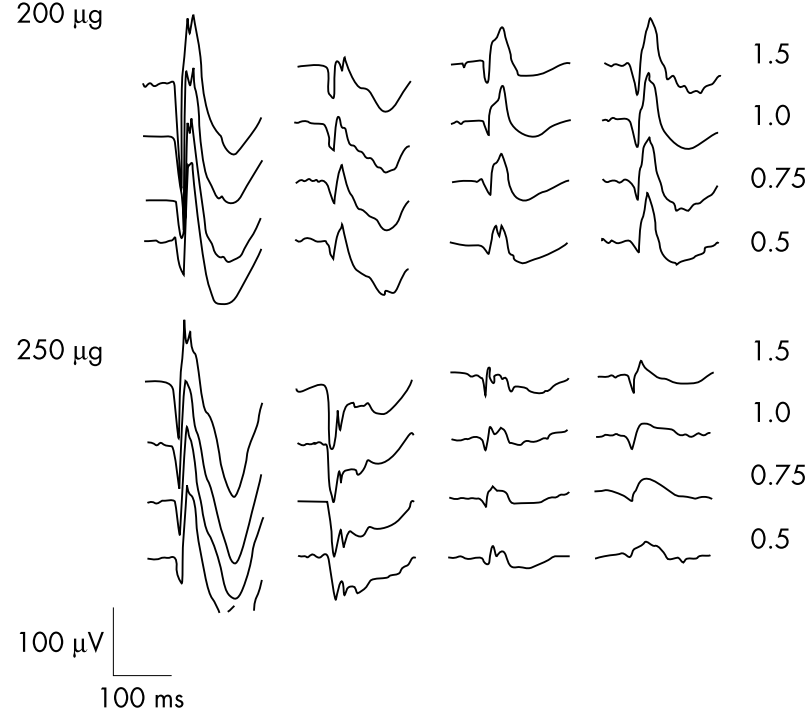

1.5

1.0

Figure 1 Typical ERG recordings of rabbit eyes before, 1 day, 1 week, and 2 months after exposure to $50,150,200$, and $250 \mu$ tenecteplase. Results from all four strobe intensities $(0.5,0.75,1.0$, and 1.5) are presented for each time point. Scale bar shows $100 \mu \mathrm{V}$ in the $y$-axis and $100 \mathrm{~ms}$ in the $\mathrm{x}$-axis.

injected with $50 \mu \mathrm{g}$, one eye was excluded because of retinal touch during injection. One of the remaining eyes had a hypopyon and mild vitritis on day 1 which resolved after a week, and was thought to be the result of iatrogenic lens touch. Another eye had a mild vitritis on day 1 which again had resolved by 1 week. The remaining five eyes injected with $50 \mu \mathrm{g}$ tenecteplase showed no evidence of anterior or posterior chamber inflammation. The eye injected with $100 \mu \mathrm{g}$ tenecteplase showed no signs of anterior or posterior chamber inflammation.

None of the five eyes injected with $150 \mu \mathrm{g}$ tenecteplase showed any sign of anterior or posterior chamber inflammation. One of the eyes had a preretinal haemorrhage inferior to the disc at day 1 but this was resolved at 1 week.

Four of six eyes injected with $200 \mu \mathrm{g}$ showed moderate vitritis at day 1 . Of these four eyes, two eyes progressed to tractional retinal detachment and in one eye areas of retinal atrophy developed inferior and superior to the optic disc. The third eye with initial vitritis had normal fundal examination at 2 months. One eye was ophthalmoscopically normal at all examinations and one rabbit died before the 2 month follow up. There was no anterior chamber inflammation visible in any of the eyes. 
The most severe toxicity was seen ophthalmoscopically in the eyes injected with 250 and $350 \mu$ g tenecteplase. All of the eyes developed vitritis at day 1 . Four of the eyes also had fibrous vitreal condensations and two eyes had areas of retinal oedema. At 1 week, four eyes progressed to traction retinal detachments and three had areas of retinal necrosis. The remainder had fibrous vitreal bands. At 2 months the areas of tractional retinal detachment had increased, one eye had also developed an area of retinal atrophy, two eyes had areas of retinal necrosis, and four had fibrous traction within the vitreous.

In the eyes with retinal pathology, the area affected to the greatest extent was around the site of injection-that is, inferior to the optic disc.

\section{Vehicle injections}

The eyes injected with the vehicle equivalent of the doses above showed no anterior or posterior chamber reaction at doses up to $100 \mu \mathrm{g}$ equivalent. The $200 \mu \mathrm{g}$ dose initially showed no sign of inflammation but at the 2 month follow up, vitreal fibrosis and an area of retinal atrophy were apparent inferior to the optic disc. With the $250 \mu \mathrm{g}$ and $350 \mu \mathrm{g}$ doses, vitritis was present from day 1 and both eyes developed traction retinal detachments at 2 months.

\section{Intraocular pressure}

There was no statistical difference in intraocular pressures between control eyes and eyes receiving injections of tenecteplase at any of the doses tested (table 1).

\section{Electroretinography}

Before injection the average B-wave amplitude was 158 (8.1) $\mu \mathrm{V}$ at $0.5 \log$ stimulus intensity; $167(8.6) \mu \mathrm{V}$ at 0.75 log; 177 (9.1) $\mu \mathrm{V}$ at $1.0 \mathrm{log}$; and 190 (9.5) $\mu \mathrm{V}$ at $1.5 \log$ stimulus intensities. There was no significant reduction in Bwave amplitude with $50 \mu$ g tenecteplase $(p>0.2)$ (table 2). At $150 \mu \mathrm{g}$ tenecteplase, however, there was a drop in B-wave amplitude to $75.6 \%$ of original value by day $\mathrm{l}(\mathrm{p}<0.01)$. The drop was even more severe at higher doses of 200 and $250 \mu \mathrm{g}$ where only $51 \%$ and $39.6 \%$ of the original B-wave amplitude was seen 1 day after injection $(\mathrm{p}<0.01)$ (fig 2$)$. Although this drop recovered by 1 week in the $150 \mu$ group, only partial recovery was seen in the $200 \mu \mathrm{g}$ group to $68 \%(\mathrm{p}<0.01)$ by
2 months, and no recovery was seen in the $250 \mu$ group ( $40.1 \%$ of original B-wave amplitude at 2 months, $\mathrm{p}<0.01$ ). Regarding the doses of tenecteplase with limited data points and the eyes tested with vehicle equivalent doses, B-wave amplitude showed no reduction at $25 \mu \mathrm{g}$ but at doses of $100 \mu \mathrm{g}$ and above, there was a dose dependent reduction in amplitude.

\section{Histology}

Light microscopic examination correlated to ophthalmoscopic and electroretinographic findings and revealed the presence of visible damage to the retina with higher doses (200 and $250 \mu \mathrm{g}$ ) of tenecteplase. In most of these eyes, damage was predominantly in the inferior retina, medullary ray, and optic disc. The eyes that received 25, 50 (fig 3A), $100 \mu \mathrm{g}$ tenecteplase and 25, 50, and $100 \mu \mathrm{g}$ vehicle were indistinguishable from their respective control eyes, which appeared normal. The retinae of all these eyes had healthy looking cells in well organised layers.

However, in the eyes that received $150 \mu \mathrm{g}$ tenecteplase, minor changes in the inner nuclear layer were observed. Slight swelling of some cells close to the outer plexiform layer was seen (fig 3B). Other layers of the retina appeared normal.

In the eyes that received $200 \mu \mathrm{g}$ tenecteplase, significant retinal damage was seen in most regions of the inferior retina in all the animals. In the affected areas the retina had lost most of its layered structure and was reduced in thickness. The inner limiting membrane was intact. However, the inner nuclear layer had undergone post-necrotic changes resulting in atrophy of its cells. Vacuoles were also present. Photoreceptor cells had also atrophied and outer and inner segments were absent. Pigment epithelial cells appeared lean and scanty with loss or reduction of its lipid globules (fig 3C).

All the eyes that received $250 \mu \mathrm{g}$ tenecteplase (fig 3D) showed more severe damage than those eyes that received $200 \mu \mathrm{g}$ tenecteplase. Post-necrotic atrophy of retinal layers causing extreme reduction in thickness with infiltration of chronic inflammatory cells were noted. The changes were seen in most areas of the inferior retina. In these areas, although the inner limiting membrane was intact, large number of red blood cells and chronic inflammatory cells were present in the vitreous. The cells in the different layers of the retina were greatly reduced with a loss of inner and

Table 1 Effect of dose and duration of intravitreal tenecteplase on intraocular pressure

\begin{tabular}{llll}
\hline & \multicolumn{4}{l}{ Mean IOP (SE) (mm Hg), post injection } \\
\cline { 2 - 4 } Tenecteplase dose $(\boldsymbol{\mu g})$ & $\mathbf{1}$ Day & 1 Week & 2 Months \\
\hline 0 & $15.03(0.75)$ & $15.23(0.63)$ & $17.14(0.76)$ \\
50 & $14.57(1.29)$ & $14.85(2.78)$ & $14.43(2.12)$ \\
150 & $11.00(2.81)$ & $14.80(1.64)$ & $16.40(2.08)$ \\
200 & $10.50(1.35)$ & $13.83(0.44)$ & $15.40(1.15)$ \\
250 & $14.00(2.93)$ & $12.29(1.47)$ & $13.86(1.91)$ \\
\hline
\end{tabular}

Table 2 Ophthalmoscopic, electroretinographic, and histological findings with increasing doses of intravitreal tenecteplase

\begin{tabular}{|c|c|c|c|c|}
\hline & \multicolumn{4}{|c|}{ Tenecteplase dose $(\mu \mathrm{g})$} \\
\hline & $50(n=7)$ & $150(n=5)$ & $200(n=5)$ & $250(n=7)$ \\
\hline Ophthalmoscopy & Normal & Normal & $\begin{array}{l}\operatorname{TRD}(2) \\
\operatorname{RA}(1) \\
\text { Normal(2) }\end{array}$ & $\begin{array}{l}\operatorname{TRD}(3) \\
\operatorname{RN}(2) \\
\operatorname{VF}(2)\end{array}$ \\
\hline $\begin{array}{l}\text { ERG B-wave amplitude } \\
\text { Retinal histology }\end{array}$ & $\begin{array}{l}\text { No reduction } \\
\text { Normal }\end{array}$ & $\begin{array}{l}\text { Transitory reduction } \\
\text { Mild damage }\end{array}$ & $\begin{array}{l}\text { Significant reduction } \\
\text { Significant damage }\end{array}$ & $\begin{array}{l}\text { Significant reduction } \\
\text { Significant damage }\end{array}$ \\
\hline
\end{tabular}

TRD = tractional retinal detachment; RA = retinal atrophy; $\mathrm{RN}=$ retinal necrosis; $\mathrm{VF}=$ vitreal fibrosis. 


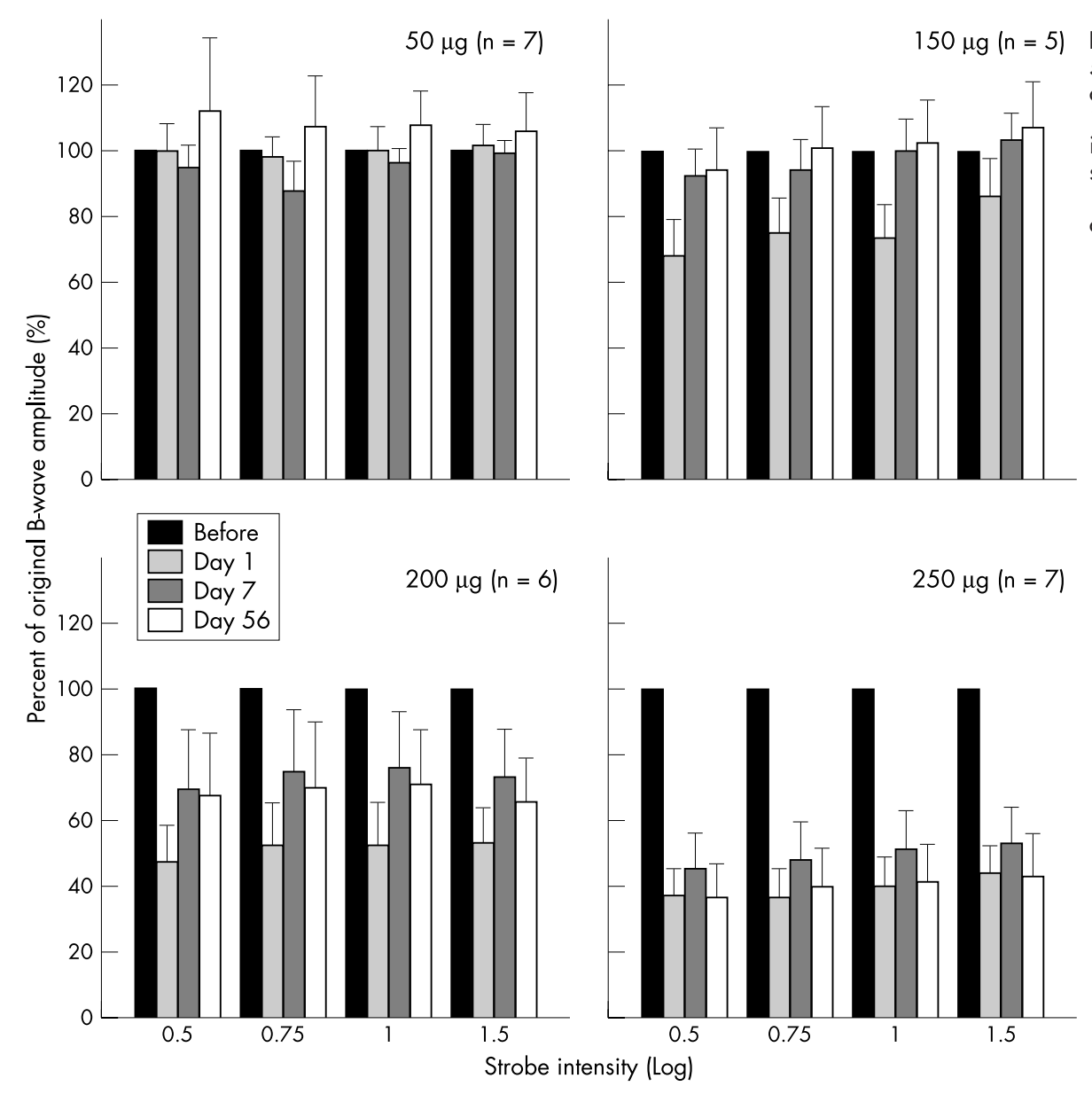

Figure 2 Graph showing the effect of $50,150,200$, and $250 \mu \mathrm{g}$ tenecteplase on B-wave amplitude measured before, 1 day, 1 week, and 2 months after the injection. B-wave amplitude at all stimulus intensities $(0.5,0.75,1.0$, and 1.5) are presented as a percentage of original B-wave amplitude.

outer retina including rod inner and outer segments and vacuoles were present. The lamellar architecture of the retina was completely destroyed and replaced by glial cell proliferation. Chronic inflammatory cells in the retina were also seen. Pigment epithelial cells had shrunk or extruded lipid globules vacuolation and appeared to be undergoing necrosis.

The most severe cases were in the eyes that received the $250 \mu \mathrm{g}$ and $350 \mu \mathrm{g}$ vehicle, in which there was full thickness necrosis with the loss of most of the retina except for a remnant thin strand of cells.

\section{DISCUSSION}

Tissue plasminogen activator (tPA) has been widely used in the treatment of acute myocardial infarction but because of the short half life of tPA its administration requires a bolus injection followed by a two step 90 minute infusion. ${ }^{7}$ Newer thrombolytic agents such as tenecteplase have been developed to improve on the characteristics of tPA by substituting three single amino acids at the $\mathrm{T}, \mathrm{N}$, and $\mathrm{K}$ domains. ${ }^{10}$ This has resulted in a compound with a longer half life in the systemic circulation (18 minutes compared to 4 minutes) enabling bolus administration, potentially improving the lytic rate by exposing the clot to a rapid high concentration of enzyme. $^{71011}$ Tenecteplase also has 14 times the fibrin specificity of tPA, which theoretically improves potency and allows enzymatic activity to occur preferentially at the clot rather than in the peripheral circulation reducing the risk of systemic fibrinolysis. ${ }^{710}{ }^{11}$ Tenecteplase is also 80 times more resistant to inactivation by plasminogen activator inhibitor-1 (PAI-1), an enzyme secreted by platelets that inhibits thrombolytics. $^{71011}$
In the field of ophthalmology, tPA has various indications for use, the most common being displacement of submacular haemorrhage. Given the potential advantages of tenecteplase over tPA, tenecteplase may have greater efficacy in the management of this condition and also in the management of central retinal vein occlusion. However, the retinal toxicity profile of tenecteplase needs to be established. The toxicity of tPA has previously been studied in the rabbit and $\mathrm{cat}^{89}$ and established that evidence of retinal toxicity occurred with intravitreal doses greater than or equal to $50 \mu \mathrm{g}$. Thus, our data suggest that tenecteplase is less toxic to the rabbit eye than tPA. In human eyes, a dose of $25-50 \mu \mathrm{g}$ has been commonly used clinically ${ }^{5}{ }^{12-14}$ but exudative retinal detachment has occurred with a dose of $100 \mu \mathrm{g}^{13}$ and fundus pigmentary change with a dose of $33 \mu \mathrm{g} .{ }^{15}$ The toxicity of tPA is thought to be due to the L-arginine component of the vehicle as vehicle equivalents of the tPA doses used in the above work caused identical retinal toxicity. ${ }^{8}$ Further evidence comes from a study of intravitreal injections of aztreonam (also containing L-arginine in the vehicle), which showed almost equivalent retinal damage when a vehicle equivalent to the aztreonam dose was injected. ${ }^{16}$

Our study has demonstrated no signs of tenecteplase induced retinal toxicity at intravitreal doses up to and including $50 \mu \mathrm{g}$.

There was no sign of tenecteplase induced toxicity in fundus appearance at tenecteplase doses up to $150 \mu \mathrm{g}$. However, the ERG showed transient suppression at this dose at day 1 post-treatment. Histopathological examination revealed minor changes in the inner nuclear layer mainly in the cells close to the outer plexiform layer. 
A
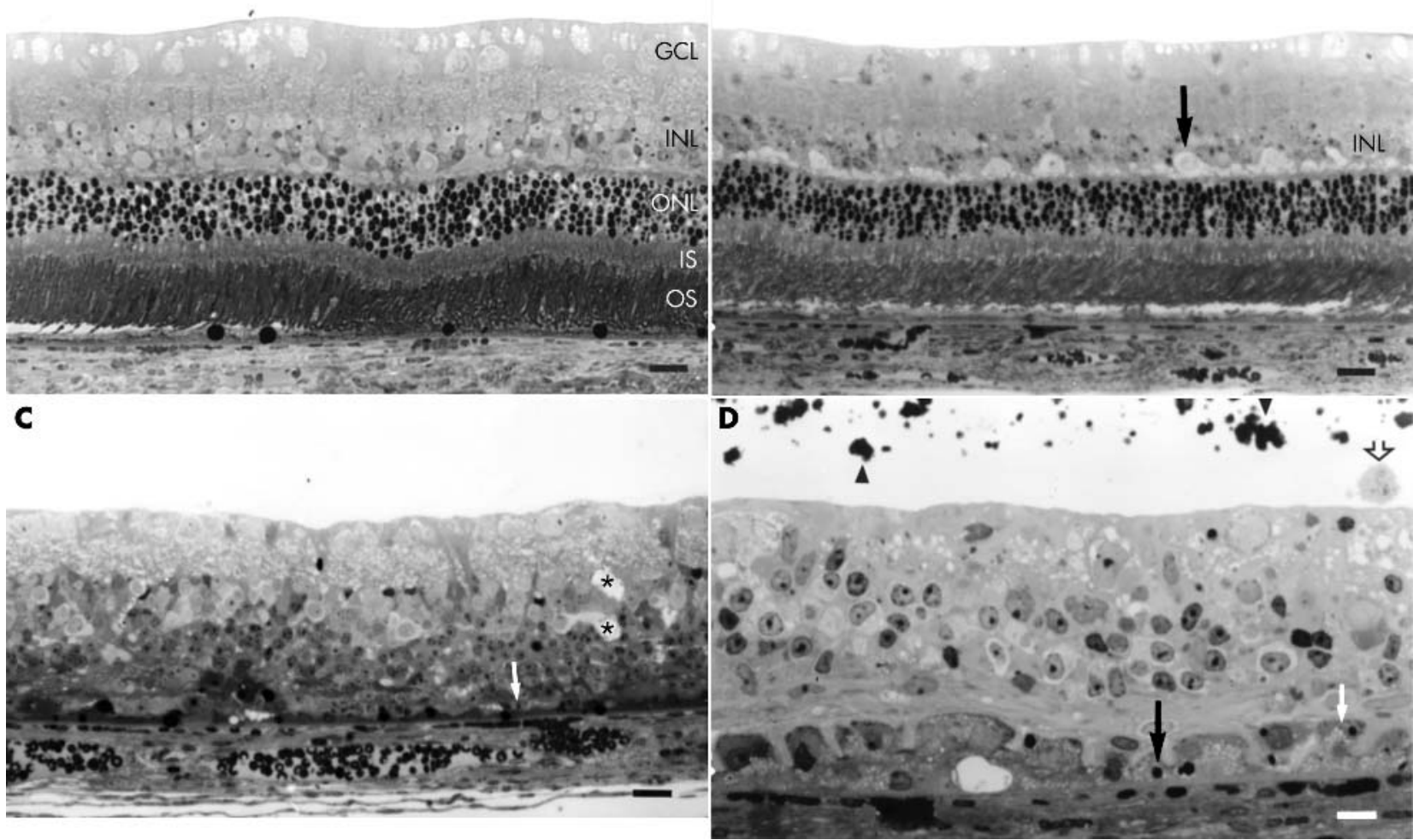

Figure 3 (A) Light micrograph of a retina, 2 months after intravitreal injection of $50 \mu \mathrm{g}$ tenecteplase in $0.1 \mathrm{ml}$ balanced salt solution. Retinal architecture of all layers of the retina including rod outer (OS) and inner segments (IS), outer nuclear layer (ONL), inner nuclear layer (INL) and ganglion cell layer (GCL) appear to be normal (stained with toluidine blue, magnification, scale bar $=20 \mu \mathrm{m}$ ). (B) Light micrograph of a retina, 2 months after intravitreal injection of $150 \mu \mathrm{g}$ tenecteplase in $0.1 \mathrm{ml}$ balanced salt solution. Mild swelling of cells in the inner nuclear layer (INL) can be seen (arrow). Other layers of the retina appear to be normal (stained with tolvidine blue, magnification, scale bar $=20 \mu \mathrm{m}$ ). (C) Light micrograph of a retina, 2 months after intravitreal injection of $200 \mu \mathrm{g}$ tenecteplase in $0.1 \mathrm{ml}$ balanced salt solution. Significant damage consisting of pigment epithelial cell necrosis (white arrow), post-necrotic atrophy of all layers of the neural retina including rod outer and inner segments, outer nuclear layer, inner nuclear layer and ganglion cell layer causing reduction in thickness. Vacuoles are also seen (*) (stained with toluidine blue, magnification, scale $\mathrm{bar}=20 \mu \mathrm{m}$ ). (D) Light micrograph of a retina, 2 months after intravitreal injection of $250 \mu \mathrm{g}$ tenecteplase in $0.1 \mathrm{ml}$ balanced salt solution. Severe damage of the retina with pigment epithelial cell necrosis and localised proliferation (white arrow) with shrunken lipid globules (arrow) is seen. Postnecrotic atrophy of all layers of the neural retina including rod outer and inner segments outer nuclear layer, inner nuclear layer, and ganglion cell layer causing thinning and gliosis is also seen. Degenerated red blood cells (arrowhead) and a macrophage (open arrow) in the vitreous are present (stained with toluidine blue, magnification, scale bar $=10 \mu \mathrm{m}$ ).

At a dose of $200 \mu \mathrm{g}$ tenecteplase, significant reductions of B-wave amplitude were noted and histopathological examination revealed significant retinal damage in most regions of the inferior retina in all eyes. There was necrosis of pigment epithelial and retinal cells, post-necrotic atrophy of cells causing thinning of the retina, and gliosis. Despite these ERG and histopathological findings, some eyes displayed no ophthalmoscopic evidence of retinal toxicity.

With tenecteplase doses of 250 and $350 \mu \mathrm{g}$ the ophthalmoscopic evidence of retinal damage was more severe with the majority of the inferior retina being affected. This was confirmed histopathologically, with damage occurring distant to the site of injection and also on ERG with the greatest reductions in B-wave amplitude being in this group.

Although the vehicle components for commercially available tPA are similar to that of tenecteplase, the concentration is significantly different. A vial containing $10 \mathrm{mg}$ tPA contains $348 \mathrm{mg}$ of L-arginine-that is, $34.8 \mathrm{mg}$ L-arginine/ $1.0 \mathrm{mg}$ tPA, whereas a vial containing $40 \mathrm{mg}$ of tenecteplase has $417.6 \mathrm{mg}$ of L-arginine-that is, $10.44 \mathrm{mg}$ L-arginine/ $1.0 \mathrm{mg}$ tenecteplase (product information, Boehringer Ingelheim). Thus for equivalent doses, tenecteplase has less than one third of the L-arginine content than with tPA. We found that the toxicity profile of the vehicle alone was identical to the equivalent tenecteplase dose. This gives further evidence that the retinal toxicity of these compounds is primarily caused by the vehicle.

Although we have found that $50 \mu \mathrm{g}$ tenecteplase injected intravitreally causes no evidence of retinal toxicity, it should be noted that our study was performed on phakic rabbits with formed vitreous. The rabbit retina is predominantly avascular and the vitreous volume is smaller than that of humans, so it is difficult to extrapolate these results for tenecteplase use in humans. The structurally similar tPA however has shown the same toxicity profile in cats and rabbits, despite the cat having a larger vitreous volume and a vascularised retina ${ }^{89}$ and the use of intravitreal and subretinal tPA at $50 \mu \mathrm{g} / 0.1 \mathrm{ml}$ has shown no signs of toxicity in humans. ${ }^{12-14}$ Given that tenecteplase contains less Larginine dose for dose compared with tPA and has the same molecular weight, one would expect that $50 \mu \mathrm{g}$ tenecteplase would not cause retinal toxicity in humans.

Other unanswered questions at present are whether the theoretical advantages of tenecteplase over tPA translate into greater clinical efficacy in the resolution of submacular haemorrhage and whether its increased potency will cause problems such as increased rates of re-bleeding. In vitro studies showed a 7.5-fold increase in potency of tenecteplase 
compared with that of tPA with respect to lysis of whole blood clots in rabbit arterial venous shunts. ${ }^{10}$ Clinical studies showed no significant difference in coronary artery reperfusion rates of tenecteplase and $\mathrm{tPA}^{17}$ and a large multicentre trial (ASSENT-2) showed equivalence of tenecteplase and tPA in 30 day mortality rates in the treatment of acute myocardial infarction. ${ }^{18}$ Regarding the potential risk of rebleeding, the ASSENT-2 trial found similar rates of intracranial haemorrhage and significantly less non-cerebral bleeding events in the tenecteplase group compared to tPA. ${ }^{18}$

In summary, we have demonstrated that intravitreal injection of a novel fibrinolytic, tenecteplase, results in dose dependent retinal toxicity in phakic, non-vitrectomised rabbit eyes. There were no signs of retinal toxicity at doses up to and including $50 \mu \mathrm{g}$ tenecteplase. Further work is under way to determine if tenecteplase has the potential for greater efficacy in the treatment of submacular haemorrhage and central retinal vein occlusion.

\section{ACKNOWLEDGEMENTS}

The authors would like to thank Judy Granger for laboratory assistance. Grant support was provided by the National Health and Medical Research Council of Australia, grant number 211901.

\section{Authors' affiliations}

S A Rowley, S Vijayasekaran, P K Yu, I L McAllister, D-Yi Yu, Centre for Ophthalmology and Visual Science, The University of Western Australia, Perth, Western Australia, Australia

\section{REFERENCES}

1 Wedrich A, Menapace R, Ries E, et al. Intracameral tissue plasminogen activator to treat severe fibrinous effusion after cataract surgery. $J$ Cataract Refract Surg 1997;23:873-7.

2 Jaffe GJ, Abrams GW, Williams GA, et al. Tissue plasminogen activator for postvitrectomy fibrin formation. Ophthalmology 1990;97:184-9.
3 Weiss JN, Bynoe LA. Injection of tissue plasminogen activator into a branch retinal vein in eyes with central retinal vein occlusion. Ophthalmology 2001; 108:2249-57.

4 Elman MJ. Thrombolytic therapy for central retinal vein occlusion: results of a pilot study. Trans Am Ophthalmol Soc 1996;94:471-504.

5 Johnson MW. Pneumatic displacement of submacular hemorrhage. Curr Opin Ophthalmol 2000;11:201-6.

6 Tanswell P, Tebbe U, Neuhaus KL, et al. Pharmacokinetics and fibrin specificity of alteplase during accelerated infusions in acute myocardial infarction. J Am Coll Cardiol 1992;19:1071-5.

7 Davydov L, Cheng JW. Tenecteplase: a review. Clin Ther 2001 ;23:982-97.

8 Johnson MW, Olsen KR, Hernandez E, et al. Retinal toxicity of recombinant tissue plasminogen activator in the rabbit. Ophthalmology 1990; 108:259-63.

9 Hrach CJ, Johnson MW, Hassan AS, et al. Retinal toxicity of commercial intravitreal tissue plasminogen activator solution in cat eyes. Arch Ophthalmol 2000;118:659-63.

10 Keyt BA, Paoni NF, Refino CJ, et al. A faster-acting and more potent form of tissue plasminogen activator. Proc Natl Acad Sci USA 1994;91:3670-4.

11 Gibson CM, Marble SJ. Issues in the assessment of the safety and efficacy of tenecteplase (TNK-tPA). Clin Cardiol 2001;24:577-84

12 Haupert CL, McCuen BW, Jaffe GJ, et al. Pars plana vitrectomy, subretinal injection of tissue plasminogen activator, and fluid-gas exchange for displacement of thick submacular hemorrhage in age-related macular degeneration. Am J Ophthalmol 2001;131:208-15.

13 Hesse L, Schroeder B, Heller G, et al. Quantitative effect of intravitreally injected tissue plasminogen activator and gas on subretinal hemorrhage. Retina 2000;20:500-5.

14 Hattenbach LO, Klais C, Koch FH, et al. Intravitreous injection of tissue plasminogen activator and gas in the treatment of submacular hemorrhage under various conditions. Ophthalmology 2001;108:1485-92.

15 LaPuente CR, Peyman GA, et al. Guidelines for intravitreal treatment of vascular occlusions with riPA (abstract). Invest Ophthalmol Vis Sci 1998;39(suppl):988.

16 Loewenstein A, Zemel E, Lazar M, et al. Drug-induced retinal toxicity in albino rabbits: the effects of imipenem and aztreonam. Invest Ophthalmol Vis Sci 1993;34:3466-76.

17 Cannon CP, Gibson CM, McCabe CH, et al. TNK-tissue plasminogen activator compared with front-loaded alteplase in acute myocardial infarction: results of the TIMI 10B trial. Thrombolysis in Myocardial Infarction (TIMI) 10B Investigators. Circulation 1998;98:2805-14.

18 Assessment of the Safety and Efficacy of a New Thrombolytic. Single-bolus tenecteplase compared with front-loaded alteplase in acute myocardial infarction: the ASSENT-2 double-blind randomised trial. Lancet 1999:354:716-22. 